Revue des patrimoines

43 | 2021

Des écoles d'art académiques aux écoles d'art : des collections et des lieux, un patrimoine à valoriser

\title{
Patrimoine muséal et éducation artistique en Italie autour de 1882
}

La séparation des galeries d'art des académies des beaux-arts

Museum heritage and artistic education in Italy around 1882: the separation of art galleries from the academies of Fine Arts

La separazione delle pinacoteche dalle accademie di Belle Arti. Patrimonio

museale e istruzione artistica in Italia intorno al 1882

\section{Martina Lerda}

Traducteur : Marie Caillat

\section{(2) OpenEdition \\ Journals}

Édition électronique

URL : http://journals.openedition.org/insitu/29128

DOI : 10.4000/insitu.29128

ISSN : $1630-7305$

Éditeur

Ministère de la Culture

Référence électronique

Martina Lerda, «Patrimoine muséal et éducation artistique en Italie autour de 1882 », In Situ [En ligne],

43 | 2021, mis en ligne le 22 janvier 2021, consulté le 22 janvier 2021. URL : http://

journals.openedition.org/insitu/29128; DOI : https://doi.org/10.4000/insitu.29128

Ce document a été généré automatiquement le 22 janvier 2021.

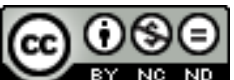

In Situ Revues des patrimoines est mis à disposition selon les termes de la licence Creative Commons Attribution - Pas d'Utilisation Commerciale - Pas de Modification 4.0 International. 


\section{Patrimoine muséal et éducation artistique en Italie autour de 1882}

La séparation des galeries d'art des académies des beaux-arts

Museum heritage and artistic education in Italy around 1882: the separation of art galleries from the academies of Fine Arts

La separazione delle pinacoteche dalle accademie di Belle Arti. Patrimonio

museale e istruzione artistica in Italia intorno al 1882

Martina Lerda

Traduction : Marie Caillat

\section{NOTE DE L'AUTEUR}

Cette contribution est issue de ma thèse de doctorat, « Le pinacoteche statali nell'Italia unita. Politiche museali e affermazione della disciplina storico-artistica (1859-1882)

[Les pinacothèques de l'État dans le royaume d'Italie : politiques muséales et affirmation de la discipline historico-artistique (1859-1882)] », soutenue en 2015 à l'Università degli Studi di Udine. Je remercie sincèrement Donata Levi et Simona Rinaldi pour leurs précieuses indications.

1 Au lendemain de la proclamation du Royaume d'Italie (1861), le paysage des musées d'État, sous la tutelle du ministère de l'Instruction publique, se compose de collections ayant appartenu à diverses institutions publiques héritées des États pré-unitaires et des collections formées aux siècles précédents par les anciennes dynasties tout récemment déchues. La configuration institutionnelle de cet immense patrimoine est le résultat des dynamiques de nationalisation des collections mises en œuvre par les gouvernements provisoires qui, entre 1859 et 1860, ont immédiatement précédé, dans les régions de la Péninsule, l'annexion au Royaume d'Italie.

2 Au terme de ce processus, seules quelques illustres collections de peintures se constituent en pinacothèques autonomes, directement placées sous la tutelle du 
ministère de l'Instruction publique : les galeries royales florentines (la Galleria degli Uffizi et celle du palais Pitti, qui devient publique en juin $1859^{1}$ ) et, à Turin, la pinacothèque de la Maison de Savoie qui, en 1860, est nationalisée sur le modèle toscan ${ }^{2}$ - ce qui était loin d'aller de soi. À Naples, la collection de tableaux des Bourbons, composée d'œuvres provenant des palais de Capodimonte et de Francavilla, était quant à elle réunie depuis 1806, avec d'autres collections "dynastiques ", au Palazzo degli Studi, pour constituer une collection secondaire dans un musée essentiellement consacré à la conservation de vestiges antiques. Le gouvernement dictatorial de Garibaldi le déclare Musée national en décembre $1860^{3}$. Le destin de la collection des ducs d'Este, conservée au Palais ducal de Modène, est encore différent : elle est mise à la disposition de l'académie des Beaux-Arts locale en $1859^{4}$. Comme dans le cas antérieur de la petite collection de tableaux du Palais ducal de Lucques ${ }^{5}$, la collection de Modène est intégrée aux collections historiques dépendant des institutions d'enseignement de l'État. Parmi elles, on compte la pinacothèque palermitaine, annexée aux collections archéologiques de l'Université jusqu'en $1863^{6}$, et les pinacothèques des académies des Beaux-Arts de Milan, Bologne, Parme, Florence, auxquelles s'ajoute, à partir de 1866 et l'annexion de la Vénétie au Royaume d'Italie, celle de Venise.

3 Ainsi, à partir de cette reconstruction complexe, au moment de la proclamation de l'unité de l'Italie, on voit que la majeure partie des collections de tableaux historiques de l'État se trouvent annexées aux académies de Beaux-Arts et sont donc considérées comme des instruments connexes à l'enseignement artistique.

4 Le décret du 13 mars 1882, qui sépare du point de vue administratif les collections historiques des académies des Beaux-Arts, intervient de manière décisive sur la structure du patrimoine muséal d'État : il soustrait les pinacothèques des académies et les transforme en institutions autonomes, en d'autres termes directement placées sous la tutelle du ministère de l'Instruction publique ${ }^{7}$. Cette réforme, encore peu étudiée, est loin d'être marginale puisqu'elle a contribué à déterminer la physionomie du système muséal moderne italien et a eu des répercussions sur le lien fort entre collections picturales et formation artistique que le Royaume d'Italie avait hérité des États préunitaires et des gouvernements de transition.

5 Il ne s'agit pas ici de reconstruire l'histoire de chacune des collections académiques italiennes mais plutôt de se concentrer sur le processus d'élaboration et la signification de cette mesure décisive.

6 Si cette réforme représente sans aucun doute un changement de nature administrative, on ne peut pas nier qu'elle eut aussi des effets sur le plan culturel. L'enjeu, en effet, réside ici dans la fonction assignée par la sphère politique aux pinacothèques historiques qui, à partir de ce moment, cessent d'être comprises comme des instruments connexes à l'enseignement artistique et voient leur vocation officielle étendue. Il faut considérer ce nouvel état de fait comme le résultat d'un processus complexe, dans lequel agissent des mouvements de différentes natures: des revendications disciplinaires des premiers défenseurs du connoisseurship italien aux tentatives tourmentées de réforme des académies des Beaux-Arts, jusqu'à l'exigence de l'État italien naissant de doter son système muséal d'une organisation administrative.

7 Tout d'abord, la place centrale des pinacothèques dans la culture des connaisseurs du $\mathrm{XIX}^{\mathrm{e}}$ siècle permet de réfléchir aux rapports qui existent entre élaboration institutionnelle et instances issues des milieux les plus à la pointe de la critique historico-artistique. En d'autres termes, l'étude des politiques relatives aux 
pinacothèques, comme celle-ci, fournit la possibilité d'observer le processus d'affirmation de l'histoire de l'art comme discipline autonome en Italie depuis une perspective originale. Les dynamiques impliquées dans l'élaboration de cette mesure sont donc analysées ici à partir des contributions de quelques connaisseurs célèbres en particulier Giovanni Morelli et Giuseppe Mongeri - à la définition d'une fonction muséale étendue des collections de peinture italiennes. Leur contribution vise notamment à l'introduction de critères de classement cohérents (mouvement qui peut s'inscrire dans le cadre décrit par Krzysztof Pomian quant à l'entrée de l'histoire au musée $\left.{ }^{8}\right)$ mais aussi à la professionnalisation et à la reconnaissance des compétences historico-artistiques nécessaires à l'administration des collections de peinture. C'est dans un tel contexte que se forment les premières propositions pour l'autonomie des pinacothèques.

\section{L'autonomie des pinacothèques d'après les propositions des connaisseurs}

8 Le milieu des connaisseurs trouve dans les pinacothèques un instrument fondamental pour l'affirmation de la discipline historico-artistique naissante. Ainsi apparaissent, dès les premières années du Royaume d'Italie, quelques propositions de réorganisation des collections, compatibles avec les modèles historiographiques les plus récents. Il suffit de rappeler à ce propos deux célèbres interventions publiques : le discours tenu par Giovanni Morelli à la Chambre en juillet $1862^{9}$ et l'article de Giovanni Battista Cavalcaselle, publié en 1863 dans la Rivista dei Comuni italiani ${ }^{10}$. Ces deux interventions dénoncent à l'unisson la difficile situation des pinacothèques italiennes au lendemain de l'unité italienne et présentent des solutions adaptées, comme la vérification des attributions, la mise à jour des catalogues, l'achèvement et le reclassement cohérent des collections. Sur ce dernier point, Cavalcaselle se prononce en faveur du critère chronologique par école de peinture, plus adapté à la traduction visuelle des développements de l'histoire de l'art, selon les méthodes de recherche adoptées par le connaisseurship européen.

9 Cette revendication disciplinaire des connaisseurs se confronte cependant, inévitablement, à l'autorité traditionnelle exercée par les académies de Beaux-Arts dans le domaine de la conservation. En effet, en diverses occasions, les critiques appuyées de Cavalcaselle n'épargnent pas le travail effectué par ces institutions, qui "démontrent leur peu de savoir sur l'art ancien, ou feignent de ne pas savoir, ou ne s'en soucient guère ${ }^{11} »$. Toutefois, c'est Morelli qui, le premier, ouvre concrètement le débat sur le rôle des académies des Beaux-Arts dans la gestion du patrimoine muséal. Dans un projet de loi du début des années 1860, qui fut abandonné, Morelli propose précisément la séparation des pinacothèques des académies des Beaux-Arts, à partir du présupposé suivant: "L'art ancien réclame absolument d'être séparé de l'art moderne $^{12}$.»

10 La distinction entre la fonction de conservation et l'enseignement artistique implique en effet, fondamentalement, de reconnaître de nouvelles compétences professionnelles, et vise au dépassement de ce qu'un autre connaisseur, Giuseppe Mongeri, définissait comme le "préjugé trop commun qui conduit à faire l'amalgame entre l'artiste et le connaisseur de l'art ancien ${ }^{13} »$. En outre, la création d'institutions placées sous la tutelle directe du ministère permettrait enfin de refondre la structure des pinacothèques sur 
le modèle des plus grands musées européens. C'est ce qui apparaît dans une nouvelle proposition rédigée, une fois encore, par Morelli et Mongeri en février $1870^{14}$. Ici, l'autonomie des pinacothèques constitue un postulat fondamental aussi bien pour l'adoption de principes homogènes dans la gestion des institutions de l'État que pour une redéfinition radicale du système muséal national tout entier.

11 Les deux connaisseurs imaginent en effet une grande pinacothèque à Florence, qui représenterait de manière synthétique les différentes écoles italiennes. Aux institutions dispersées sur le territoire national, ils assignent la tâche d'illustrer les développements des différentes écoles de peinture locales. Dans cette optique, Morelli et Mongeri légitiment les échanges, les centralisations et les recompositions d'œuvres démembrées. L'hypothèse d'un tel système muséal semble ainsi avoir tendance à négliger la stratification historique des collections; en revanche, elle se révèle certainement propice à l'affirmation et à la diffusion de la discipline historicoartistique.

Le ministre de l'Instruction publique Cesare Correnti, culturellement et politiquement proche des deux connaisseurs, approuve les lignes directrices du projet, au point de présenter certaines orientations de la réforme dans le cadre des mesures de rééquilibrage budgétaire. L'article 8 de l'annexe $C$, présenté à la Chambre le 10 mars 1870, prévoit le transfert des académies des Beaux-Arts aux communes. L'article 9 , quant à lui, établit que: "Les pinacothèques et les collections artistiques et archéologiques appartenant à l'État, mais aussi celles qui sont unies aux académies et aux écoles des Beaux-Arts, seront constituées et administrées séparément de toute autre institution et seront réorganisées de manière à ce que chacune d'entre elles représente, de façon aussi complète que possible, les écoles d'art et les mémoires historiques du pays à l'endroit où elle se situe ${ }^{15}$. »

13 La réaction du milieu académique à ce projet est immédiate, et donne lieu à un débat explicite et passionnant sur la fonction des collections de tableaux anciens.

\section{Le rôle des pinacothèques : deux visions en regard}

Quelques jours après la présentation du projet, le Conseil académique milanais transmet une motion de contestation au ministre et aux autres académies des BeauxArts du royaume. Si la principale objection polémique des académiciens milanais est la dépendance communale éventuelle de l'institution, d'âpres critiques concernant l'hypothèse de l'autonomie de la pinacothèque de Brera se font également entendre. Deux motifs sont opposés à cette hypothèse: l'importance pédagogique de la pinacothèque et la connexion naturelle entre la collection et l'académie milanaises. En effet, on peut lire dans le document que "la séparation de la pinacothèque de l'académie soustrairait à la libre action du corps enseignant l'un des instruments les plus efficaces de son ministère ${ }^{16} »$. Enfin, dans la lettre qui accompagne la motion, le président de l'académie milanaise, Carlo Belgiojoso, s'oppose à une réorganisation rigoureuse des collections sur le critère de l'école locale, qui mettrait en péril l'intégrité de la pinacothèque de Brera, née comme une collection de chefs-d'œuvre. Elle serait alors, en effet, contrainte de céder des œuvres d'art exceptionnelles, comme le Mariage de la Vierge de Raphaël, en échange de morceaux d'une histoire picturale déjà amplement représentée à l'extérieur de l'institution. 

constituent-elles pas d'ores et déjà le fonds essentiel grâce auquel elles [les pinacothèques] peuvent se vanter de leur richesse ? " À la lumière de cet argument, la disposition défendue serait conforme à la cohérence naturelle des collections. Pour atteindre cet objectif, il suffirait simplement que des conservateurs ayant acquis des compétences spécifiques révisent les attributions, sélectionnent les œuvres, examinent 
les pièces conservées dans les réserves ou en dépôt dans les églises. Et enfin, qu'ils s'accordent sur d'éventuels échanges avec d'autres institutions.

21 C'est précisément ce dernier point qui fait craindre, parmi quelques "victimes inconscientes de cet amour du patrimoine familial », la perspective dramatique « de les voir battre les tableaux des pinacothèques italiennes comme s'il s'agissait d'un jeu de cartes pour les répartir ensuite en groupes selon les signes dont ils portent la marque ». Cependant, étant donné que les permutations résulteraient d'accords entre les institutions elles-mêmes, selon Mongeri, l'idée que, par exemple, le Mariage de la Vierge de Raphaël puisse se retrouver à Florence ou à Pérouse, comme le craignent les académiciens milanais, est invraisemblable. Néanmoins, une telle atténuation de ses hypothèses les plus radicales ne signifie pas pour autant que Mongeri adhère à la sensibilité moderne pour la sédimentation historique des collections, qui est un concept encore lointain. Dans sa logique, en effet, une réévaluation globale des expériences, même mineures, des différentes écoles, justifierait de mettre de côté les craintes d'une dénaturation des collections.

Entre-temps, la fonction des pinacothèques, évoquée par l'élaboration institutionnelle, devient un sujet de débat également au sein du milieu académique. Le second Congrès artistique, réunissant des académiciens, des artistes et des critiques, qui eut lieu à Milan en 1872, prévoyait en effet une session consacrée à l'archéologie artistique, dont l'enjeu était de discuter, entre autres choses, des critères d'aménagement et de catalogage des musées. Certains aspects de la discussion promus par le comité d'organisation du Congrès reprennent à la lettre les positions de Mongeri et Belgiojoso (membres eux-mêmes du comité ainsi que de la commission en charge du choix des thèmes) : ainsi, on s'y demande notamment si, à travers l'agencement des œuvres, « il serait préférable de mettre en évidence les analogies et donc de se concentrer avant tout sur les écoles et les œuvres locales ou plutôt de montrer les variétés et les contrastes qui doivent être distingués ${ }^{20} »$. Cette fois, l'assemblée nationale d'experts soutient, avec quelque prudence, le classement chronologique par écoles de peinture, sans pour autant aller jusqu'à appuyer l'idée d'une représentation de l'école de peinture locale ${ }^{21}$.

23 En ce qui concerne le débat sur l'enseignement, le Congrès réfléchit également au rôle du patrimoine historico-artistique dans la formation des jeunes artistes. Dans le cadre d'un débat entre classicisme et rénovation naturaliste, l'assemblée discute la primauté de l'étude des œuvres du passé sur l'étude d'après nature. Une certaine ambiguïté demeure cependant au sujet de la signification à attribuer au terme «étudier ». Parmi les différentes positions qui émergent, il semble que l'étude de l'antique soit communément comprise comme copie et imitation. Au terme de la discussion, cependant, l'assemblée approuve un ordre du jour présenté par l'écrivain et critique d'art Enrico Panzacchi dans lequel on recommande non pas l'étude mais l'observation des œuvres du passéz ${ }^{2}$. Une distinction terminologique subtile mais essentielle, comme Camillo Boito, l'un des signataires de la motion, le souligne ${ }^{23}$.

24 Il semble donc que les pinacothèques, de réserves de modèles qu'elles étaient, soient alors en train de devenir, également pour les artistes, des lieux de médiation sur les dynamiques historico-artistiques. À tel point qu'en 1882, c'est la Commission permanente des beaux-arts elle-même - organe consultatif ministériel composé entièrement d'artistes et pour moitié expression directe des académies des Beaux-Arts 
- qui promeut l'autonomie des pinacothèques dans une proposition (ensuite abandonnée) de restructuration de l'enseignement artistique ${ }^{24}$.

\section{L'autonomie des pinacothèques, entre réforme des académies et réorganisation ministérielle}

collections : la réforme institutionnelle des académies des Beaux-Arts. Entre 1869 et 1870, le Conseil des beaux-arts [Giunta di Belle Arti], organe consultatif du ministère de l'Instruction publique, travaille à un projet de statut général qui, en quelques années, inspire les réformes de chacune des académies du Royaume ${ }^{25}$. Ce projet, en quelques mots, vise à moderniser l'enseignement artistique conformément à ce qui émerge du débat du secteur, mais surtout à imposer un contrôle direct et centré sur le fonctionnement des écoles, en érodant l'autorité des milieux académiques locaux. La nouveauté la plus importante, en effet, est la distinction, à l'intérieur des académies des Beaux-Arts, entre collèges académiques et instituts : ces derniers, qui représentent la composante pédagogique, sont alors placés sous la tutelle directe du ministère de l'Instruction publique.

projet de statuts ne spécifie pas clairement le destin des pinacothèques. Du côté de l'application de la réforme, néanmoins, si l'on met de côté le cas de la collection qui devient propriété de l'Accademia di San Luca romaine ${ }^{26}$, c'est l'attribution aux instituts qui est généralement privilégiée, bien qu'elle comporte quelques ambiguïtés qui nécessitent des éclaircissements officiels à plusieurs reprises, comme la pinacothèque de Parme, dont le directeur de l'institut, Francesco Scaramuzza, va jusqu'à demander, sans succès, l'autonomie administrative ${ }^{27}$.

L'affectation généralisée des collections aux instituts marque une rupture avec l'attribution traditionnelle de l'expertise à l'académie mais confirme la fonction pédagogique des collections de peinture. Finalement, la réforme des académies a surtout des effets indirects ou collatéraux sur la configuration des collections.

L'autonomie concrète des pinacothèques historiques ne résulte donc pas des élans des connaisseurs en faveur d'une extension de la fonction des collections et pour la reconnaissance des compétences historico-artistiques nécessaires à leur gestion, ni d'une réflexion institutionnelle sur le rapport entre patrimoine historico-artistique et enseignement artistique. Il faut plutôt situer l'élan décisif dans l'exigence d'une rationalisation administrative.

L'autonomie des pinacothèques est en effet conçue comme la condition nécessaire pour organiser de manière uniforme les personnels impliqués dans la gestion des collections de l'État, qui étaient jusque-là dépendants à divers titres du ministère de l'Instruction publique. Le 13 mars 1882, en même temps que le décret établissant la séparation des collections des institutions d'enseignement, on publie également le premier Ruolo unico del personale addetto a Musei, alle Gallerie, agli Scavi e ai Monumenti nazionali ${ }^{28}$ ("Rôle unique du personnel chargé des musées, des galeries, des fouilles et des monuments nationaux »). En outre, il faut noter la manière dont cette nouvelle configuration simplifie la gestion directe, par le ministère, des recettes issues du droit d'entrée, introduit aussi dans les pinacothèques de beaux-arts par le décret royal de $1875^{29}$. 


\section{Conclusions} on l'a vu, de complexes motivations d'ordre culturel, leur séparation effective des académies se concrétise avant tout sous l'effet d'exigences de nature administrative. En outre, au départ, le changement est uniquement nominal. Les collections sont, à tous égards, maintenues sous l'influence académique, conservent leur siège propre (le transfert de la pinacothèque de Modène, déjà physiquement séparée de l'institution académique, vers son nouveau siège, au Palazzo dei Musei, a lieu en 1894) et le rôle de directeur est attribué à des professeurs ou à d'anciens membres du corps académique ${ }^{30}$. Mais ce sont les décrets royaux de 1882 qui permettent la confirmation successive à la direction des galeries de personnalités comme Corrado Ricci et Giulio Cantalamessa, spécialistes auxquels on doit la mise en œuvre de la mise à jour scientifique des pinacothèques. Une minute ministérielle de 1896, qu'il faut attribuer à la plume d'Adolfo Venturi, le fondateur de l'histoire de l'art comme discipline universitaire en Italie $^{31}$, fait l'éloge des aspects positifs de la réforme, aussi bien sur le plan purement administratif que sur le plan scientifique :

Sur le plan administratif, cet arrêté royal a été très utile car il a déterminé l'existence des galeries et des musées médiévaux et modernes, qui ne possédaient ni catalogues de leurs propres collections, ni normes générales et fixes pour régler leur fonctionnement. Sur le plan scientifique, ces institutions ont vu le jour, elles se sont développées selon un plan rationnel, et elles se sont organisées selon des critères que la science définit progressivement ${ }^{32}$.

31 Pour Venturi, finalement, le décret royal de 1882 a conduit à la "résurgence d'institutions qui ne donnaient plus signe de vie». Si les académies des Beaux-Arts «conservaient» les collections «en y voyant seulement un instrument pour l'enseignement ", désormais, transformées en véritables " archives de la civilisation ", les pinacothèques répondent enfin aux attentes de la "culture nationale» et de l'« histoire de la civilisation ». Et l'on peut même aller jusqu'à dire qu'elles ont accru leur utilité pédagogique, puisqu'en "accroissant leur propre patrimoine, et en le conservant avec un soin particulier, elles concourent aussi à l'objectif des académies ». Venturi ne manque pas, en conclusion, de souligner la portée disciplinaire et professionnelle de la réforme : "La division du travail a marqué la frontière entre le domaine de l'artiste qui produit et celui du spécialiste qui recherche et analyse. Une telle séparation devait se refléter également dans ces institutions, afin qu'elles puissent rencontrer leurs fins sociales, et elle a trouvé en Italie plus tard qu'à l'étranger sa traduction ${ }^{33}$.»

Les bénéfices administratifs, scientifiques et professionnels liés à l'autonomie des pinacothèques historiques ne font aucun doute. On trouve en revanche des lectures divergentes de la complexe déconnexion entre l'institution académique et le monde de la protection du patrimoine culturel. Commentant la réforme du ministre Pasquale Villari qui, en 1891, séparait les services ministériels compétents en matière d'art contemporain et d'art ancien, le spécialiste Andrea Emiliani observe à quel point « au moment où les académies des Beaux-Arts achèveront leur traditionnelle cohabitation avec les services de la conservation et de la muséographie (1891), le monde de l'unité des arts semblera à jamais révolu ». On perd ainsi, selon lui, le « contact avec le poids spécifique et la matérialité des choses de l'art. Et avec lui, encore, ce qui rapprochait 
l'acte de la création de sa contrepartie critique, qui unissait l'invention moderne à la méditation historique ${ }^{34}$.»

\section{ANNEXES}

\section{Abréviations pour les sources d'archives}

AABAF = Archivio dell'Accademia di Belle Arti di Firenze

ACS, MPI, AA. BB. AA. = Archivio Centrale dello Stato, Ministero della Pubblica Istruzione, Direzione Generale Antichità e Belle Arti

ACS, MPI, CSPI = Archivio Centrale dello Stato, Ministero della Pubblica Istruzione, Consiglio Superiore della Pubblica Istruzione

\section{NOTES}

1. Décret du commissaire extraordinaire Carlo Boncompagni en date du 21 juin 1859, reproduit dans S. N., Atti e documenti editi e inediti del Governo della Toscana. Parte prima, Florence, s. n., 1860, p. 244-245.

2. Loi du 24 juin $1860, \mathrm{n}^{\circ} 4135$.

3. Décret du dictateur des Deux-Siciles Giuseppe Garibaldi en date du 12 septembre 1860, reproduit dans S.N., Atti del governo estratti dal Giornale Ufficiale di Napoli, $\mathrm{n}^{\circ} 3,1860$ (13-17 septembre), p. 42.

4. Décret du dictateur Farini en date du 22 avril 1859, reproduit dans BOLLATI Emmanuele, Fasti legislativi e parlamentari delle rivoluzioni italiane nel secolo XIX, vol. 2, 1859-1860, Lombardia-Emilia, Milan, Giuseppe Civelli, 1865, p. 583.

5. La collection, exposée au Palais ducal, est composée de pièces provenant des galeries royales florentines, concédées à la demande du grand-duc Léopold de Habsbourg-Toscane (Léopold II) autour de 1850 et confiées au président de la commission de Promotion et de l'institut des BeauxArts. La collection reste sous la tutelle de l'institut des Beaux-Arts jusqu'en 1875, date à laquelle elle devient propriété communale.

6. En 1863, les collections de l'Université de Palerme passent sous l'autorité de la Commission des antiquités et des beaux-arts. Les collections restent dans le palais de l'université jusqu'à ce que, suite à leur accroissement dû aux suppressions des ordres et des congrégations religieuses en 1866, le musée royal soit déplacé dans un lieu plus spacieux, dans l'ancien couvent des Pères philippins de l'Olivella. En 1873, l'institut acquiert le titre de Musée national.

7. «Les galeries, les pinacothèques et les musées archéologiques, annexés à l'Université, aux académies ou aux instituts des Beaux-Arts, cesseront de faire partie desdits instituts scientifiques et artistiques, et possèderont une administration propre, dont les employés exerceront le rôle unique de personnel tel que défini par Notre autre Décret publié à la même date [Le Gallerie, le Pinacoteche e i Musei archeologici, annessi alle Università, alle Accademie e Istituti di belle arti, cesseranno di far parte dei detti Istituti scientifici od artistici, ed avranno un'amministrazione propria, con impiegati compresi nel ruolo unico del personale stabilito con altro Nostro Decreto di pari data] », décret royal en 
date du 13 mars 1882, n 678, "qui sépare les galeries, les pinacothèques et les musées des instituts d'Enseignement [che separa dagli Istituti d'insegnamento le Gallerie, le Pinacoteche ed i Musei]».

8. POMIAN Krzysztof, «Le musée face à l'histoire ", in S. N., L'Histoire au musée, actes du colloque éponyme (Versailles, château de Versailles, 10-12 décembre 1998), Arles, Actes Sud, 2004, p. 99-126.

9. Son discours est publié en annexe à DALAI EMILIANI Marisa, «Giovanni Morelli e la questione del Catalogo Nazionale: un episodio poco noto della politica di tutela nell'Italia dell'Unità ", in AGOSTI Giacomo, MANCA Maria Elisabetta \& PANZERI Matteo (dir.), Giovanni Morelli e la cultura dei conoscitori, actes de colloque (Bergame, 1987), Bergame, P. Lubrina, 1993, p. 123-128.

10. CAVALCASELLE Giovanni Battista, "Sulla conservazione dei monumenti e degli oggetti di belle arti e sulla riforma dell'insegnamento accademico. G. B. Cavalcaselle al signor Ministro della pubblica istruzione ", Rivista dei Comuni italiani, 1863 ; repris dans Accademia Clementina. Atti e memorie, 1987, $\mathrm{n}^{\text {os }}$ 20-21, p. 85-112. Pour une analyse de ce document, voir LEVI Donata, Cavalcaselle. Il pioniere della conservazione dell'arte italiana, Turin, G. Einaudi, 1988, p. 309-322; EMILIANI Andrea, «Giovanni Battista Cavalcaselle politico. La conoscenza, la tutela e la politica dell'arte negli anni dell'unificazione italiana », in TOMMASI Anna Chiara (dir.), Giovanni Battista Cavalcaselle conoscitore e conservatore, Venise, Marsilio, 1998, p. 323-369.

11. " [M]ostrano di saper poco dell'arte antica, o fingono di non sapere, o non si occupano " (Giovanni Battista Cavalcaselle au ministre de l'Instruction publique Carlo Matteucci, le 24 août 1862). Cité dans LEVI Donata, «Storiografia artistica e politica di tutela. Due memorie di G.B. Cavalcaselle sulla conservazione dei monumenti (1862) », in LA BARBERA Simonetta (dir.), Gioacchino Di Marzo e la critica d'arte nell'Ottocento in Italia, actes de colloque (Palerme, 2003), Bagheria, Aiello \& Provenzano, 2004, p. 74.

12. "L'arte antica vuole assolutamente essere segregata dall'arte moderna" (Giovanni Morelli à Gaetano Giordani, le 28 juillet 1862). Cité dans CAMMAROTA Gian Piero, Le Origini della Pinacoteca Nazionale di Bologna. Una raccolta di fonti, vol. 2, Dalla rifondazione all'autonomia (1815-1907), Bologne, Minerva, 2004, p. 417.

13. "[Il] pregiudizio troppo comune che mette a fascio l'artista coll'intendente dell'arte antica»: MONGERI Giuseppe, «Le belle arti davanti al Parlamento italiano », La Perseveranza, n 966, 24 juillet 1862, p. 3.

14. «Proposte d'ordinamento per le Pinacoteche e i Musei Nazionali », 9 février 1870, dans ACS, MPI, AA. BB. AA., I versamento, « Musei, Gallerie e Pinacoteche », b. 170, fasc. 1-1.

15. "Le pinacoteche e le raccolte artistiche ed archeologiche appartenenti allo Stato, ed ancor quelle che sono unite alle accademie e scuole di belle arti, verranno costituite e amministrate separatamente da ogni altro istituto e saranno riordinate in modo che ciascuna di esse rappresenti, più compiutamente che sia possibile, le scuole d'arte e le memorie storiche del paese ove è collocata. " Cité dans Atti parlamentari, Camera dei Deputati [Chambre des députés]. X Legislatura, session 1869-1870, Documenti, $\mathrm{n}^{\circ} 53$, Provvedimenti pel pareggio del bilancio presentati dal Ministro delle Finanze (Sella) nella tornata del 10 marzo 1870, Allegato C, Legge sull'istruzione pubblica, art. 9, p. 172.

16. «[L]a separazione della Pinacoteca dalla Accademia sottrarrebbe alla libera azione del Corpo insegnante uno dei più efficaci istrumenti del suo ministero " (le président de l'académie des BeauxArts de Milan, Carlo Belgiojoso, au président de l'académie des Beaux-Arts de Florence, Niccolò Antinori, le 12 avril 1870, dans AABAF, filza 1870).

17. "[N]uocerebbe alla diffusione della cultura artistica»; "proficui apprezzamenti che porge la comparazione di opere di scuole diverse » (ibid.)

18. "[D]are ad esse [le pinacoteche] un colore tutto locale accogliendovi i soli capo-lavori di ogni singola scuola danneggerebbe gli studii togliendo la possibilità dei confronti; offenderebbe il carattere universale che l'arte dee avere; le farebbe perdere quelle nuove qualità, che le impartirono le tolte barriere, la 
comunanza e la fratellanza delle varie famiglie italiane " (Délibération du conseil académique de Venise, le 18 avril 1870, dans ACS, MPI, AA. BB. AA., «Istituti di belle arti », b. 6).

19. MONGERI Giuseppe, "Dell'ordinamento delle pubbliche pinacoteche in Italia ", Nuova Antologia, vol.17, $\mathrm{n}^{\circ}$ 5, 1871, p. 57-78.

20. «[C]onvenga meglio mettere in evidenza le analogie e quindi, occuparsi a preferenza delle scuole e delle opere locali, ovvero mostrare le varietà ed $i$ contrasti onde vanno contraddistinte » : REALE ACCADEMIA DELLE BELLE ARTI DI MILANO, Atti del secondo Congresso artistico italiano a Milano nel settembre 1872, Milan, A. Lombardi, 1874, p. 15.

21. «On a aussi abordé le meilleur moyen d'organiser les pinacothèques : et bien que l'on reconnaisse qu'elles doivent généralement servir la nature des locaux, la grandeur des peintures et les dispositions précédentes, on a considéré que la distribution par écoles, et à l'intérieur de celles-ci par époques, était souhaitable. [Si toccò pure del miglior modo di ordinare le pinacoteche: $e$ pur riconoscendo ch'esse generalmente devono servire alla natura dei locali, alla grandezza dei dipinti e alle disposizione precedenti, si trovò desiderabile la distribuzione per scuole, equeste per età]: REALE ACCADEMIA DELLE BELLE ARTI DI MILANO, Atti del secondo Congresso..., p. 190.

22. "Les soussignés estiment que lorsqu'un jeune atteint le niveau de pouvoir dessiner à partir du relief les formes des objets en général, avec une exactitude satisfaisante et une bonne intelligence des logiques de la perspective et du clair-obscur, [...] il devrait passer à l'étude d'après nature ; désignant par nature les moulages en plâtres tirés d'après nature, les drapés, le modèle vivant, le paysage, etc. Mais reconnaissant dans le même temps qu'il est hautement souhaitable que les exemples de bons artistes soient toujours exposés à la vue des étudiants aux fins de comparaisons utiles, ils proposent l'ordre du jour suivant: pour les jeunes, l'étude et l'imitation de la nature, et l'observation des œuvres des bons artistes doivent aller de pair. I sottoscritti opinano che quando un giovane sia giunto al grado di poter ritrarre dal rilievo le forme degli oggetti in genere, con sufficiente esattezza e con giusta intelligenza delle ragioni della prospettiva e del chiaroscuro, [...] debba passare anzi tutto allo studio del vero; intendendo per vero i gessi ricavati dal naturale, i panneggiamenti, il modello dal vivo, il paese ecc. Ma riconoscendo in pari tempo essere sommamente opportuno che gli esempi dei buoni artisti stiano sempre esposti alla vista degli studiosi per utili confronti, propongono il seguente ordine del giorno: Che i giovani debbano fare camminare di pari passo lo studio e l'imitazione del vero, colla osservazione delle opere dei buoni artisti.]»: REALE ACCADEMIA DELLE BELLE ARTI DI MILANO, Atti del secondo Congresso..., p. 103.

23. " [N]otre ordre du jour dit "observer" les œuvres des grands maîtres et non pas "étudier", celui de M. Villa dit "étudier" et non pas "observer". La différence est ici absolument essentielle et il faut la souligner, afin que l'on ne dise pas que nous avons voulu faire passer notre ordre du jour avec des malentendus [Il nostro ordine del giorno dice "osservare" le opere dei grandi maestri e non "studiare", quello del signor Villa dice "studiare" e non "osservare". La differenza qui è essenzialissima e importa farla notare, perché non si dica che abbiamo voluto far passare il nostro ordine del giorno con degli equivoci] » (ibid., p. 104).

24. Sur les travaux de la Commission, voir ACS, MPI, AA. BB. AA., « Istituti di belle arti », b. 3.

25. Deux versions du projet sont conservées: ACS, MPI, CSPI, "Giunta di Belle Arti », b. 59, fasc. 59. Pour en savoir plus sur le contexte des travaux du Conseil, voir MARZINOTTO Marica, "Giovanni Battista Cavalcaselle "nemico" della Pontificia Accademia di San Luca ", Ricerche di storia dell'arte, $\mathrm{n}^{\circ} 107,2012$, p. 47-58. Les principaux points du projet sont repris à titre expérimental dans les statuts de l'Accademia di San Luca à Rome et dans ceux de l'Accademia delle Arti e del Disegno à Florence, publiés les 9 octobre 1873 et 3 novembre 1873 (modifiés les 22 et 29 juin 1874 et le 3 décembre 1876). La réforme est ensuite étendue aux académies des BeauxArts de Modène, Bologne, Parme, avec le décret royal du 23 septembre 1877, et à l'académie des Beaux-Arts de Venise, avec le décret royal du 8 septembre 1878. Sur les réformes, voir : BOITO Camillo, «I nuovi decreti sulle accademie di belle arti », Nuova Antologia, vol. 25, $\mathrm{n}^{\circ}$ 4, 1874, p. 880-896; CARAMEL Luciano, «Le accademie artistiche italiane nel corso del XIX secolo: una 
lunga crisi di identità ", Ottocento. Catalogo dell'arte italiana dell'ottocento, $\mathrm{n}^{\circ} 18,1989$, p. 27-30 ; NICOSIA Concetto, Arte e Accademia nell'Ottocento. Evoluzione e crisi della didattica artistica, Bologne, Minerva, 2000, p. 204-209; ROCCASECCA Pietro, «Appunti per una storia della formazione accademica degli artisti (1754-1923) », in BARBIERI Costanza, The Lost Art of drawing. Disegni inediti di architettura dal Fondo storico dell'Accademia di Belle Arti di Roma, catalogue d'exposition (Rome, Centro Studi Americani, 2016), Rome, L'Erma di Bretschneider, 2016, p. 61-66.

26. L'Accademia di San Luca, privée de ses fonctions pédagogiques par le décret royal du 29 juin 1874, devient indépendante du ministère de l'Instruction publique. Le décret de 1882 ne touche donc pas à ses collections, profondément liées à l'histoire de l'institution.

27. Voir la correspondance dans ACS, MPI, AA. BB. AA., I versementi, « Musei, Gallerie e Pinacoteche ", b. 306, fasc. 169-3. Dans le cas de Venise, au contraire, l'affectation des collections à l'institut est partiellement révoquée par le décret royal du 9 novembre 1879.

28. Décret royal du 13 mars $1882, n^{\circ} 679$ (série 3), «qui approuve le rôle unique du personnel chargé des musées, des galeries, des fouilles et des monuments nationaux [che approva il ruolo unico degli impiegati addetti ai Musei, alle Gallerie, agli Scavi ed ai Monumenti Nazionali] ». Les décrets du 13 mars suivent une refonte de la répartition des compétences au niveau ministériel. À ce sujet, il faut rappeler surtout la création, en 1875, de la direction centrale des Fouilles et des Musées royaux. Ce nouveau service technique est confié à l'archéologue Giuseppe Fiorelli, maître d'œuvre d'une opération de rationalisation de la gestion des musées d'antiquités. Parmi ses interventions les plus importantes, signalons la rédaction du « Ruolo organico e Regolamento pel servizio dei musei di antichità dello Stato » («Rôle organique et règlement du service des musées nationaux d'antiquités »). Voir JALLA Daniele, Museo contemporaneo. Introduzione al nuovo sistema museale italiano, Turin, UTET, 2003, p. 65-69. Avec la création, en 1881, de la direction générale des Antiquités et des Beaux-Arts, le domaine artistique est également attribué à la direction de Fiorelli qui peut ainsi étendre sa politique de centralisation aux musées de Beaux-Arts.

29. Voir LERDA Martina, « La Politica museale dopo l'Unità. L'introduzione dell'ingresso a pagamento nei musei statali (1860-1875)», mémoire sous la direction d'Antonella Gioli et Ettore Spalletti soutenu à l'Université de Pise, 2010 ; RICCO Antonello, "Tasse e tessere d'ingresso in musei, gallerie, scavi e monumenti governativi del Regno d'Italia (1875-1939) », Aedon, n 3, 2011.

30. La fonction de directeur est occupée à Bologne par Cesare Masini, à Milan par Giuseppe Bertini, à Modène par Adeodato Malatesta, à Venise par Nicolò Borazzi et à Parme par Cecrope Barilli, en même temps directeur de l'institut des Beaux-Arts. À Florence, en revanche, la responsabilité des anciennes collections académiques est transférée à la Soprintendenza delle Regie Gallerie.

31. Voir AGOSTI Giacomo, La Nascita della storia dell'arte in Italia. Adolfo Venturi dal museo all'università 1880-1940, Venise, Marsilio, 1996 ; D’ONOFRIO Mario (dir.), Adolfo Venturi e la storia dell'arte oggi, Modène, F. C. Panini, 2008.

32. « Amministrativamente, quel Decreto Reale recò una utilità grandissima perché determinò l'esistenza delle gallerie e dei musei medievali e moderni i quali non avevano $i$ cataloghi del loro patrimonio, non generali e costanti norme per il loro funzionare. Scientificamente, quelle istituzioni presero vita, si accrebbero secondo un disegno razionale, si ordinarono secondo $i$ criteri che la scienza va determinando mano a mano" (ministère de l'Instruction publique au secrétariat d'État pour l'Instruction publique, le 7 avril 1896, dans ACS, MPI, AA. BB. AA., II versamento, III parte, b. 118).

33. «La divisione del lavoro ha segnato i termini del campo dell'artista che produce, e dello studioso che ricerca e analizza. Tale divisione doveva rispecchiarsi anche in questi istituti, affinché si adattassero ai fini sociali, e si è rispecchiata in Italia più tardi che all'Estero " (ministère de l'Instruction publique au secrétariat d'État pour l'Instruction publique, le 7 avril 1896, dans ACS, MPI, AA. BB. AA., II versamento, III parte, b. 118).

34. " [N]el momento in cui le accademie di belle arti verranno distaccate dalla tradizionale convivenza con gli uffici della conservazione e della museografia (1891), il mondo dell'unità delle arti sembrerà finito per 
sempre. [Si perde così, a suo avviso, il] contatto con il peso specifico e con la materialità delle cose d'arte. E con questo, anche ciò che avvicinava l'atto della creazione al suo risarcimento critico, che univa l'invenzione moderna alla meditazione storica » : EMILIANI Andrea, "Giovanni Battista Cavalcaselle politico...», p. 327-328.

\section{RÉSUMÉS}

En 1882, un décret royal du Royaume d'Italie a retiré aux académies des Beaux-Arts la gestion des pinacothèques historiques, qui sont devenues des instituts autonomes, dépendant directement du ministère de l'Instruction publique. Bien qu'essentiellement administrative, cette importante séparation signe officiellement un changement dans la relation entre l'art du passé et l'éducation artistique, qui se vérifie au même moment avec l'affirmation de l'histoire de l'art comme discipline indépendante. D'outils pédagogiques et réserves de modèles pour les jeunes peintres qu'elles étaient, les galeries d'art deviennent officiellement des lieux d'étude, d'observation et de méditation sur les évolutions historiques et artistiques. Cette étude retrace la dynamique complexe de ce processus dans lequel entrent en jeu des mouvements de différentes natures : des revendications disciplinaires des premiers représentants du connoisseurship italien aux tentatives tourmentées de réforme des académies des beaux-arts jusqu'à l'exigence de l'État italien naissant de doter son système muséal d'une organisation administrative.

In 1882, a royal decree of the Kingdom of Italy removed from the academies of Fine Arts the role of managing historical art galleries, which became independent institutes, directly depending on the Ministry of Public Education. Although primarily administrative, this important separation officially marked a change in the relationship between art from the past and artistic education, which was illustrated at the same time by the affirmation of art history as a distinct discipline. From the learning resources and storehouse of models for budding painters that they were, the art galleries became official places for studying, observing and reflecting on historical and artistic developments. This study traces the complex dynamics of this process, in which were involved movements of different types: from the disciplinary claims of the first exponents of the Italian connoisseurship, to the attempts to reform the academies of Fine Arts, until the emerging Italian state required an administrative reorganization of the museum system.

Nel 1882 un regio decreto del Regno d'Italia sottrae alle accademie di Belle Arti la gestione delle pinacoteche storiche, che diventano istituti autonomi, direttamente dipendenti dal Ministero della Pubblica Istruzione. Sebbene di carattere prevalentemente amministrativo, questa importante cesura sancisce ufficialmente un cambiamento nel rapporto tra arte del passato e istruzione artistica, che si verifica in significativa concomitanza con il processo di affermazione della Storia dell'Arte come disciplina autonoma. Da strumenti didattici e repertorio di modelli per i giovani pittori, le pinacoteche si vanno configurando a livello istituzionale come luoghi di studio, di osservazione e di meditazione sugli sviluppi storico-artistici.

Il saggio ripercorre le complesse dinamiche di questo processo, in cui agiscono spinte differenti: dalle rivendicazioni disciplinari dei primi conoscitori, agli interventi di riforma dell'insegnamento artistico, sino alle esigenze di riorganizzazione amministrativa del sistema museale statale. 
INDEX

Keywords : Pinacotheque, Academy of Fine Arts, Gallery of Fine Arts, Italian ministry of Public Instruction, art education, connoisseurship

Parole chiave : pinacoteca, accademie di Belle Arti, Ministero della Pubblica Istruzione, istruzione artistica, connoisseurship

Mots-clés : Pinacothèque, académie des beaux-arts, galerie d'art, ministère de l'Instruction publique, éducation artistique, connoisseurship

\section{AUTEURS}

MARTINA LERDA

Docteure en histoire de l'art

martina.lerda@alice.it 